\title{
Infectious disease epidemics in refugee camps: a retrospective analysis of UNHCR data (2009-2017)
}

\author{
Chiara Altare ${ }^{1}$, Vincent Kahi', Moise Ngwa ${ }^{3}$, Amelia Goldsmith', Heiko Hering ${ }^{2}$, Ann Burton ${ }^{2}$, Paul Spiegel ${ }^{1}$ \\ ${ }^{1}$ Centre for Humanitarian Health, Department of International Health, Johns Hopkins Bloomberg School of Public Health, Baltimore, Maryland, USA, 2 \\ United Nations High Commissioner for Refugees, Public Health and HIV department, Geneva, Switzerland, ${ }^{3}$ Department of International Health, \\ Johns Hopkins Bloomberg School of Public Health, Baltimore, Maryland, USA \\ Keywords: global health \\ https://doi.org/10.29392/joghr.3.e2019064
}

\section{Journal of Global Health Reports}

Vol. 3, 2019

\begin{abstract}
Background
The majority of deaths during conflict and displacement are due to indirect causes, specifically infectious diseases. Although the control of communicable diseases and epidemics is one of the top priorities during humanitarian crises, little has been published about epidemics in refugee camps. In this article we analyze data from the health information system managed by the United Nations High Commissioner for Refugees (UNHCR) capturing key public health information from camps. We provide insights into the epidemiological profile and overall burden of epidemics in these settings in order to inform decisions on priority interventions.
\end{abstract}

\section{Methods}

We used data from UNHCR Health Information System and conducted a descriptive analysis of outbreaks between January 2009 to July 2017 in terms of frequency, geographical distribution, duration, size, case fatality, attack rate, and type of outbreaks.

\section{Results}

A total of 364 outbreaks occurred in 21 countries, affecting 108 refugee camps. Seventy-five percent of epidemics were due to measles, cholera, meningitis; $70 \%$ of them occurred in three countries (Kenya, Chad, Thailand). Fifty percent of the camps recorded $<1$ outbreak/year, while $90 \%$ of camps experienced one or two types of diseases. Half of the outbreaks lasted less than one month and had fewer than 10 cases.

\section{Conclusions}

UNHCR and partners appear to be successfully containing infectious disease epidemics in refugee camps. Preventive measures addressing water, sanitation, hygiene and shelter conditions could nevertheless reduce the risk for water and air-borne diseases. Vaccination remains a key preventive strategy that needs to be enhanced and adapted to such mobile populations.

By the end of 2017, 68.5 million people were forced to flee their homes as a result of persecution, conflict or generalized violence. ${ }^{1}$ Of these, 40 million remained within their national borders and are internally displaced persons (IDPs), 25.4 million crossed borders and are refugees and another 3.1 million are asylum seekers.

Conflict and displacement are a recognized public health risk by concomitantly increasing population vulnerability and reducing the system's response capacity. ${ }^{2,3}$ The majority of deaths in conflict and displacement are due to indirect causes, specifically infectious diseases. ${ }^{4}$ Therefore, control of communicable diseases and epidemics has been identified as one of the top ten priorities during the emergency phase of humanitarian crises since the $90 \mathrm{~s}^{5,6}$
Factors such as overcrowding, poor water and sanitation conditions, lack of vaccination, delayed diagnosis and reduced access to treatment can lead to increased occurrence, severity and case fatality of infectious diseases. However, impact and mechanisms differ according to displacement settings. Access to affordable health services tends to be a major challenge for displaced people settling outside of camps, while persons within camps can be at higher risk of infections due to overcrowding. ${ }^{7}$ Furthermore, displaced persons are often more vulnerable to epidemics during the early stages of displacement, reflecting both the precarious conditions from which they flee and the challenges in accessing care. Over time, health status of in-camp population tends to improve, while that of out-of-camp populations varies according to different contexts. ${ }^{7}$ 
Little has been published about epidemics in refugee camps, ${ }^{8}$ despite the existence of a comprehensive routine health information system (HIS) managed by the United Nations High Commissioner for Refugees (UNHCR) capturing key public health information from camps. The analysis of such a global database has the potential to provide insights into the epidemiological profile and overall burden of epidemics in these settings in order to inform decisions on priority interventions.

This article aims to fill this gap by analyzing UNHCR's data from its global HIS ${ }^{9}$ that focuses on epidemics in refugee camps between January 2009 and July 2017. To our knowledge, this is the first analysis of such a comprehensive global database on epidemics in refugee camps.

\section{METHODS}

DATA

We used data from the UNHCR's HIS. This is a standardized tool that UNHCR and its partners have developed and implemented since 2005 to ensure evidence-based program and policy formulation, and ultimately improve the health status of displaced population; it includes routine collection of data on 10 topics: population, mortality, morbidity, inpatient and referral services, laboratory, disease control, immunization, nutrition, reproductive health and HIV/ AIDS. ${ }^{9}$

UNHCR has defined a list of diseases with outbreak potential and assigned a corresponding weekly alert threshold. ${ }^{10}$ Reaching the threshold triggers the declaration of an outbreak and its reporting. A "closed outbreak report" is prepared by UNHCR Health Officers in the affected camp when the outbreak is declared as concluded. For this specific analysis, we extracted information from the HIS that pertained to all closed outbreaks over the period January $1^{\text {st }}, 2009$ to July $31^{\text {st }} 2017$, from all refugee camps worldwide where the HIS was functioning. Variables included name of country, name of camp, number of cases, number of deaths, date of reporting. Camp population size from UNHCR population statistics was added. ${ }^{11}$

\section{DISEASES, DEFINITION AND DURATION OF OUTBREAK}

Table 1 reports the diseases included in this analysis as per UNHCR standardized list. Each disease has a standard case definition based on both clinical symptoms and laboratory results (where needed) and an alert threshold. ${ }^{12}$ The incubation period for each disease as per World Health Organization (WHO) guidance ${ }^{13}$ when available is provided as well in Table 1 . The incubation period of Hepatitis E (2-10 weeks) was applied for Acute Jaundice syndrome (AJS) as AJS is used to define suspected cases of Hepatitis E in humanitarian settings or where laboratory confirmation is unlikely. ${ }^{14-16}$ In case of multiple possible etiologies for the same condition (e.g., rotavirus, norovirus, Escherichia Coli or cholera for watery diarrhea ${ }^{17-20}$; respiratory syncytial virus (RSV), rhinovirus and adenovirus for Influenza Like Illness), ${ }^{21,22}$ the longest disease incubation period was used.

We applied UNHCR's thresholds and excluded weeks that did not meet the criteria. When a threshold was defined compared to baseline (for example, 1.5 times the baseline), we accepted the HIS entries as no baseline data were available.

An outbreak was considered as concluded if no new cases occurred for twice the upper limit of the incubation period, regardless of the disease. The duration of an outbreak was calculated in days from the day when the first case was reported to the day the last case was reported. Given that the reporting was conducted on a weekly basis, to be most conservative, we added 7 days to the end of each outbreak.

Case fatality proportion was calculated as the number of deaths over the number of cases. The attack rate was calculated as the number of cases over the camp population at the end of the year when the outbreak occurred, as the camp population size at the beginning of the outbreak was not available.

\section{ANALYSIS}

We conducted a descriptive analysis of the outbreaks, focusing on frequency, geographical distribution, duration, size, case fatality, attack rate and type of outbreaks. Analysis was conducted in Stata (version 15). ${ }^{23}$ Data were mapped using ArcGIS by ESRI, release $10.5 .^{24}$

\section{ETHICAL CONSIDERATIONS}

This study was deemed as 'non-human subject research' and exempted from ethical clearance by Johns Hopkins Institutional Review Board.

\section{RESULTS}

UNHCR's HIS is consistently in place in all sites with more than 10,000 refugees, covering $67 \%$ of the total refugee population living in camps. It was used in 16 countries and 76 refugee camps in January 2009 and 22 countries and 135 refugee camps in 2017.

A total of 364 outbreaks occurred between January 2009 and July 2017 in 21 countries, affecting 108 refugee camps (Figure 1). Three quarters (75\%) of the epidemics were due to three diseases (measles, cholera and meningitis). Twothirds (66\%) of the overall number of cases were due to measles, cholera and influenza. Seventy-one percent (71\%) of epidemics occurred in three countries (Kenya (28\%), Chad (27\%) and Thailand (16.5\%)), while $50 \%$ of the total cases were reported in Thailand (22.1\%), Kenya (17.6\%) and South Sudan (13.8\%) (Figure 2). Ifo (Kenya), Mae La (Thailand) and Hagadera (Kenya) camps reported 25 (6.9\%), 22 (6\%) and 21 (5.8\%) of the 364 epidemics, respectively.

Fifty per cent $(50 \%)$ of the camps recorded less than 5 outbreaks over the entire period of 8.5 years (ie. less than one outbreak per year). The large majority of the camps (90\%) experienced one or two types of disease outbreaks. Only two camps (Hagadera (Kenya) and Mae La (Thailand)) experienced six and seven different diseases, respectively.

Table 2 reports average duration, size, attack rate and case fatality rate (CFR) by disease. These varied depending upon the disease. The shortest outbreaks were due to Shigella (average length 12 days) and the longest were due to Hepatitis E (292 days on average). In terms of size, Po- 
Table 1. Outbreak weekly threshold and incubation period by diseases included in the analysis

\begin{tabular}{|c|c|c|}
\hline Disease/ syndrome & Outbreak weekly threshold (UNHCR) & $\begin{array}{l}\text { Incubation period } \\
\text { (WHO) }\end{array}$ \\
\hline $\begin{array}{l}\text { Acute Jaundice } \\
\text { Syndrome }\end{array}$ & NA & 2-10 weeks \\
\hline Cholera & 1 case & 2 hours to 5 days \\
\hline Dengue Fever & NA & 4-10 days \\
\hline Giardiasis & NA & $5-25$ days \\
\hline H1N1 & NA & 1-4 days \\
\hline Hepatitis E & NA & 2-10 weeks \\
\hline Influenza Like IIIness & NA & 4-6 days* \\
\hline Measles & 1 case & 10 -18 days \\
\hline Meningitis & $\begin{array}{c}5 \text { cases or } 1 \text { confirmed Meningococcus or } 5 \text { cases or } 1.5 \text { times the } \\
\text { baseline }\end{array}$ & 2-10 days \\
\hline Pertussis & NA & 6-20 days \\
\hline Poliomyelitis & 1 case & 4-35 days \\
\hline Rubella & NA & 5 days to 3 weeks \\
\hline Shigella & NA & 1-4 days \\
\hline Varicella & NA & 10-21 days \\
\hline Watery Diarrhea & 1.5 times the baseline & $1-10$ days \\
\hline
\end{tabular}

CDC - Centers for Disease Control and Prevention, WHO - World Health Organization, UNHCR - United Nations High Commissioner for Refugees

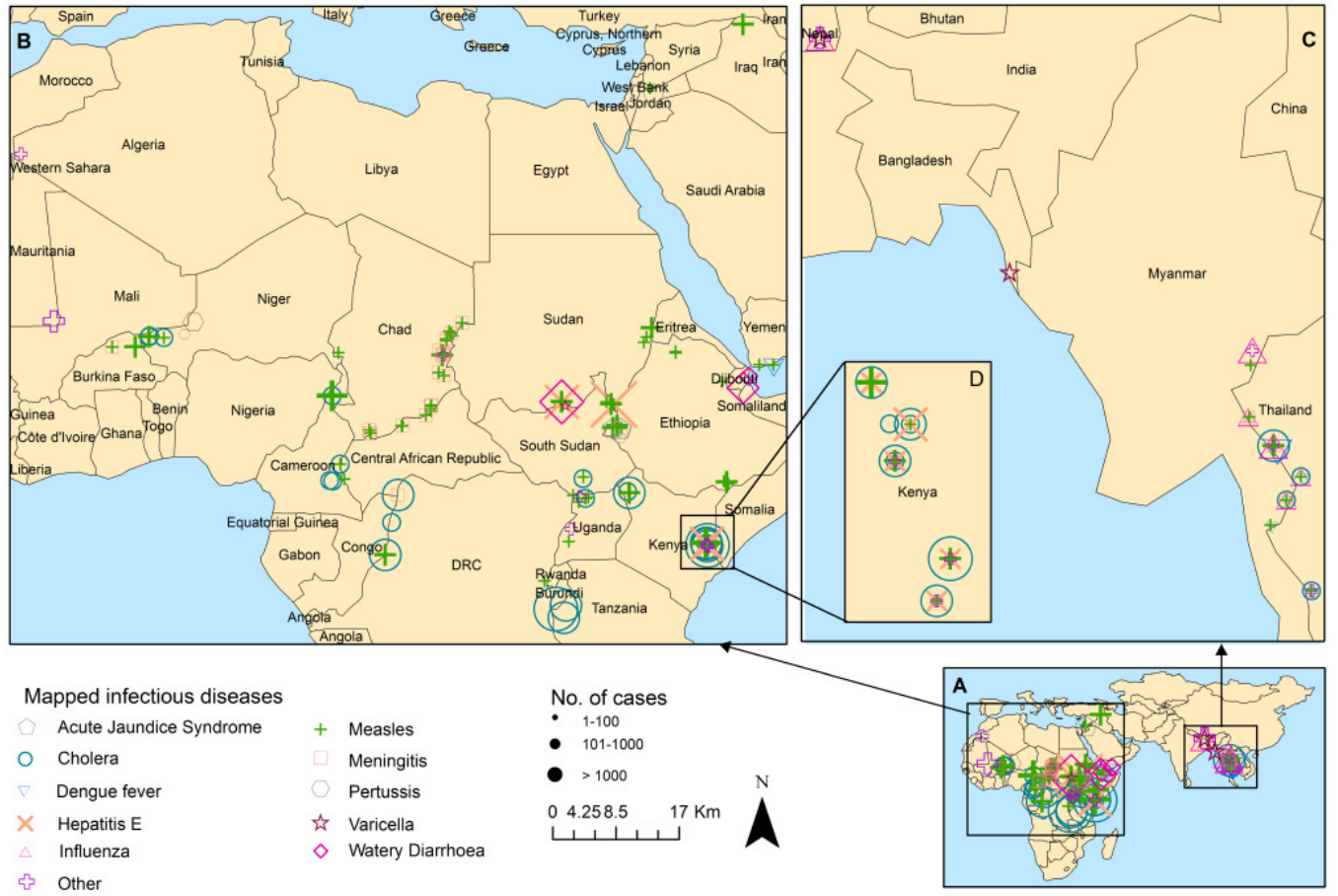

Figure 1. Worldwide case counts of infectious diseases distribution by refugee camp, 2009-2017

Note: The category "Other” includes polio, H1N1, and shigella, rubella and giardiasis. Insert A shows the burden of infectious diseases globally; inserts B and C zoom in into Africa and South East Asia; insert D shows disease occurrence in camps in Kenya.

liomyelitis and Meningitis had the smallest number of cases (5 cases on average), while the largest were due to Giardiasis, Watery Diarrhea and Influenza-like Illness (825, 674 and 552 cases on average, respectively).

The highest attack rates on average were recorded for Varicella, Influenza-like Illnesses and Watery Diarrhea. The
2009 Influenza outbreak in Beldangi I (Nepal) affected 20\% of the population, while the 2012 measles outbreak in Ayorou (Niger) affected $17 \%$. Meningitis had on average the highest CFR at 14.3\%. Median CFR for all but three diseases (Hepatitis E, AJS and Watery Diarrhea) was equal to zero. Half of the outbreaks (56\%) lasted less than one month, and 

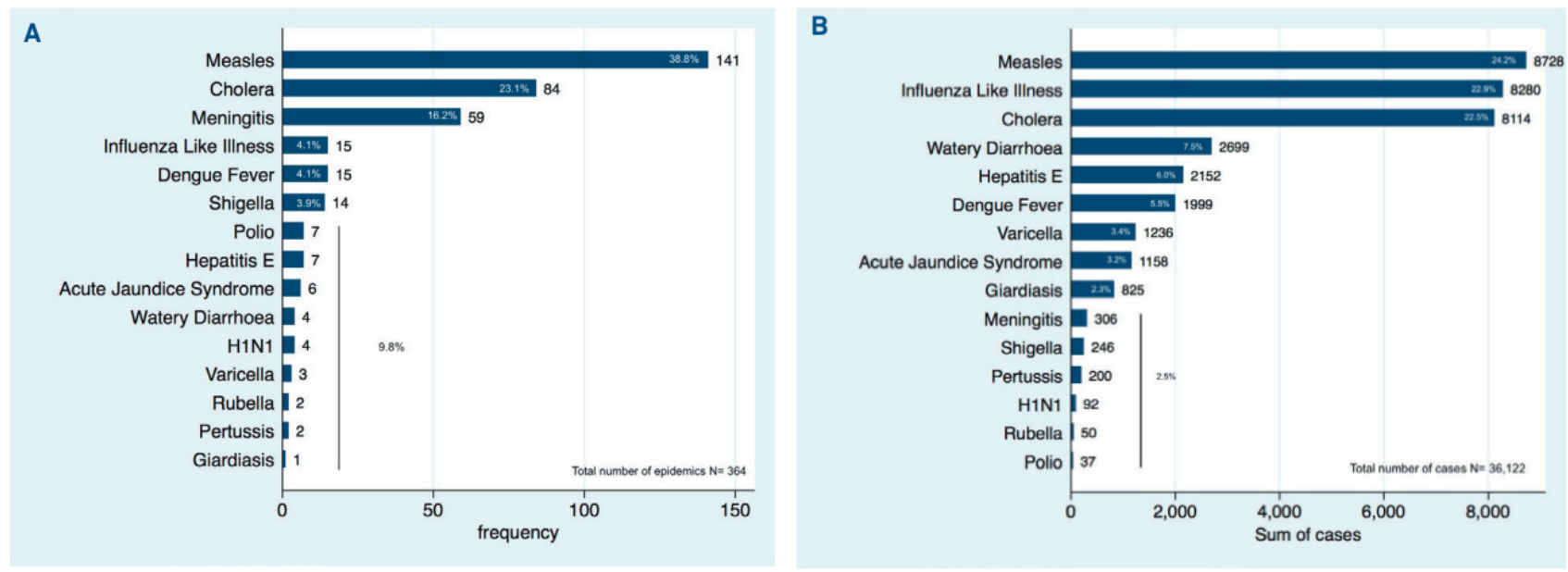

Figure 2. Frequency of infectious disease epidemics in refugee camps between 2009 and 2017

Panel A. Number of epidemics by disease. Panel B. Cumulative sum of cases by disease.

$53 \%$ had less than 10 cases. In terms of transmission routes, seven of the 15 (47\%) diseases were transmitted per fecaloral route, seven were airborne, and one was vector borne (mosquito).

\section{DISCUSSION}

This retrospective analysis of UNHCR HIS data examines epidemics that occurred in refugee camps worldwide between January 2009 and July 2017. Overall, results were encouraging: half of the camps experienced less than one outbreak per year; outbreaks tended to be rather short and small in size; and CFRs and attack rates were low. Yet, some useful lessons can be drawn from this review to strengthen infectious disease prevention in refugee camps.

The three leading diseases that caused epidemics (measles, cholera and meningitis) are vaccine-preventable; of these, only measles vaccine is provided universally in humanitarian responses and in countries' Expanded Immunization Program. The occurrence of measles outbreaks points to immunization coverage gaps. UNHCR has already expanded the measles vaccination age to include older children up to 15 years who might have never been vaccinated, ${ }^{25}$ but more systematic inclusion of adolescents and adults may be necessary in certain contexts as documented in Kenya, Tanzania and Sudan. ${ }^{26-28}$ A second challenge relates to ensuring that new arrivals are vaccinated as soon as possible, without waiting for a measles mass vaccination campaign to be organized. Enhancing mobile outreach teams and measles vaccination at screening and registration points may be a more systematic and cost-effective way to increase coverage.

The use of oral cholera vaccine (OCV) in emergencies is becoming more common despite the limited existing stockpile, the logistical capacity required, and the dearth of evidence on the most cost effective cholera intervention. ${ }^{29,30}$ WHO recommends the vaccine in endemic areas, in humanitarian crises with high risk for cholera epidemics, and during existing cholera outbreaks, always being complementary to prevention and control measures. ${ }^{31}$ In refugee camps, the focus has been on preventive measures to ensure potable water, sanitation and hygiene (WASH). ${ }^{25}$ In fact, while OCV has proven to be an effective short and medium term solution, investments in water and sanitation infrastructure together with hygiene promotion would ensure sustained and broader achievements, even beyond health. ${ }^{30}$ However, given the upfront costs of building infrastructure, as well as possible barriers to behavior change that can influence the acceptability of WASH interventions, implementation of OCV may represent the quickest and most cost-effective solution to reduce morbidity and mortality from cholera outbreaks in emergencies. Further discussion is needed as to whether OCV should be automatically provided to refugees in camps that are located in cholera prone areas, as is done for measles.

Major progress has been made in the fight against Meningitis in recent years. ${ }^{32}$ The development and the deployment of a new serogroup A meningococcal conjugate vaccine in mass vaccination campaigns as well as the expansion of vaccination country programs to include meningococcal $\mathrm{A}$ vaccine were the major contributors to this result, especially in the meningitis belt. Here, the number of cases due to serogroup A dropped dramatically. ${ }^{33}$ Displaced population need to be included in this effort, both to ensure equitable access to health services and to contribute to the global defeating of meningitis A. So far, reactive vaccination campaigns with a polysaccharide vaccine have been used to control epidemics. Similarly, as for OCV, further discussion is needed about the feasibility of routinely provide meningococcal A vaccine to refugees, at least in the meningitis belt.

The occurrence of poliomyelitis cases in numerous conflict and forced displacement settings show how vulnerable camps and host countries are to the disease until poliovirus circulation is eliminated in all countries. Polio epidemics occurred in six camps: one in Chad, two in Thailand, and three in the Dadaab complex in Kenya. Thailand and Chad each reported only one case, showing that the probable high population immunity did not allow for the virus to spread. However, the 2013 outbreak in Dadaab extended over several weeks, spread over three camps and reported 
Table 2. Characteristics of outbreaks in refugee camps, 2009-2017

\begin{tabular}{|c|c|c|c|c|c|c|c|c|c|c|c|}
\hline \multirow[t]{2}{*}{ Disease } & \multicolumn{2}{|c|}{ Length (days) } & \multicolumn{2}{|c|}{ Size (number of cases) } & \multicolumn{3}{|c|}{ Case fatality rate } & \multicolumn{3}{|c|}{ Attack rate } & \multirow{2}{*}{$\begin{array}{c}\text { Transmission route } \\
\text { type }\end{array}$} \\
\hline & mean & range & mean & range & mean & median & range & mean & median & range & \\
\hline Acute Jaundice Syndrome & 225.2 & $56-595$ & 193 & $56-487$ & 1.3 & 1.12 & $0-3.6$ & 0.4 & 0.27 & $0.1-1$ & fecal-oral \\
\hline Cholera & 36.5 & $7-372$ & 96.6 & $1-2257$ & 2.7 & 0 & $0-100$ & 0.2 & 0.02 & $0-3.1$ & fecal-oral \\
\hline Dengue Fever & 87.8 & $7-386$ & 133.3 & $1-980$ & 0.02 & 0 & $0-0.35$ & 0.4 & 0.04 & $0-2.5$ & vector borne \\
\hline Giardiasis & 112.0 & $112-112$ & 825.0 & $825-825$ & 0.00 & 0 & 00 & 1.2 & 1.24 & $1.2-1.2$ & fecal-oral \\
\hline H1N1 & 36.8 & $7-91$ & 23.0 & $4-50$ & 0.00 & 0 & 00 & 0.1 & 0.05 & $0-0.1$ & air droplet \\
\hline Hepatitis E & 292.0 & $49-833$ & 307.4 & $20-1172$ & 4.4 & 2.47 & $0-21.7$ & 0.6 & 0.27 & $0-2.3$ & fecal-oral \\
\hline Influenza Like IIIness & 112.2 & $7-413$ & 552.0 & 4-3081 & 0.1 & 0 & $0-1.8$ & 3.0 & 0.62 & $0-20.7$ & air droplet \\
\hline Measles & 70.0 & $7-630$ & 61.9 & $1-1480$ & 2.9 & 0 & $0-100$ & 0.4 & 0.04 & $0-17.5$ & air droplet \\
\hline Meningitis & 17.8 & $7-84$ & 5.2 & $1-50$ & 14.3 & 0 & $0-100$ & 0.0 & 0.01 & $0-0.2$ & air droplet \\
\hline Pertussis & 49.0 & $28-70$ & 100.0 & $85-115$ & 0.00 & 0 & 00 & 0.8 & 0.76 & $0.8-0.8$ & air droplet \\
\hline Poliomyelitis & 36.0 & $7-140$ & 5.3 & $1-25$ & 0.6 & 0 & $0-4$ & 0.0 & 0.01 & 00 & fecal-oral \\
\hline Rubella & 38.5 & $21-56$ & 25.0 & $3-47$ & 0.00 & 0 & $0-0$ & 0.1 & 0.07 & $0-0.1$ & air droplet \\
\hline Shigella & 12.6 & $7-28$ & 17.6 & $1-179$ & 0.00 & 0 & $0-0$ & 0.1 & 0.002 & $0-0.7$ & fecal-oral \\
\hline Varicella & 65.3 & $28-91$ & 412.0 & $32-805$ & 0.00 & 0 & $0-0$ & 3.3 & 2.68 & $0.2-7.2$ & air droplet \\
\hline Watery Diarrhea & 47.3 & $7-133$ & 674.8 & $13-2215$ & 0.13 & 0.02 & $0-0.4$ & 2.2 & 2.16 & $1.2-3.1$ & fecal-oral \\
\hline
\end{tabular}


34 cases. A large influx of unvaccinated Somalis into the camps, insufficient vaccination coverage of those already in the camp, and poor sanitation conditions likely contributed to the spread of the virus. Mop-up campaigns took place within days and subsequent OPV subnational campaigns within a few months. ${ }^{34}$ In such contexts, surveillance and vaccination coverage should be enhanced by strengthening screening for new arrivals and ensuring full vaccination for newborn and children, while addressing existing water and sanitation infrastructure and programs.

Three camps (Ifo (Kenya), Mae La (Thailand) and Hagadera (Kenya)) are particularly vulnerable to outbreaks, likely because of their large population sizes and numerous influx of refugees over time (over the study period, population fluctuated between 70,000 and 118,000 in Ifo; between 25,000 - 47,000 in Mae Lae; and between 71,000 and 122,000 in Hagadera). However, the attack rates show that they performed better than many smaller camps that experienced fewer outbreaks. Possibly, the fact that these camps have existed for more than 20 years (Ifo and Hagadera were established in 1991-1992 and Mae La in 1984) plays a role. They have evolved into naturally grown town with extensive health services (provided by community health workers, health posts, and referral hospitals) and general infrastructure in place that seem to be successful in containing and preventing outbreaks.

The relatively limited variability of different epidemics over time in each camp likely reflects both disease endemicity and successful prevention strategies. Most camps had to address the same type of outbreak during the period under consideration. This should pave the way for targeted interventions addressing the specific disease and transmission route. The number of diseases with a fecal oral transmission route highlight how difficult it is to achieve satisfactory WASH conditions in camps. The few studies that have been conducted in refugee camps showed that challenges exist at different levels. For example, access to water and soap alone does not guarantee actual handwashing behavior. Level of knowledge about the practice as well as alternative preferred utilization of soap (such as laundry) play a role in achieving the actual behavior. ${ }^{35,36}$ Ensuring soap availability and testing new approaches to increase awareness seem warranted. Similarly, overcrowding and poor housing conditions are a second major risk factors for transmission of diseases like measles, meningitis, and influenza. ${ }^{28,37}$ While camp design standards exist, ${ }^{25}$ underfunding of the majority of the crises, particularly over time, limit what can be achieved in many settings. Furthermore, the reluctance to consider camps as homes rather than transitory spaces hinders investments in more stable and healthier solutions. As the majority of refugees live in protracted situations outside of camp settings, ${ }^{1}$ a major challenge is to ensure safety and dignity while working to integrate refugees into host communities with the goal of improving services to both communities.

There are a number of limitations in this study. First, UNHCR HIS covers only refugee camps with a population higher than 10,000. Smaller camps and refugees living outside of camps are therefore not included. This might introduce bias in our analysis, as conditions in smaller camps might differ (although it is not straightforward how). The "closed outbreak reports" used in this analysis do not include disaggregated data by age and sex as these details are available only at camp level and are not aggregated at global level. Our analysis was therefore limited in both scope and depth.

Secondly, context might influence what is reported. Influenza-like Illness cases were recorded only in Thailand and Nepal, probably due to the increased attention on the Avian Influenza outbreak in the Asia-Pacific region at the time. However, UNHCR's standardized list of diseases that are included in the HIS limits some of this reporting bias. Context also impacts the way in which outbreaks are announced. Misclassification of particularly sensitive diseases (like cholera being labeled as acute watery diarrhea) for political reasons is therefore possible. Furthermore, declaring an outbreak as closed is context- and disease-specific. We applied the rule of doubling the incubation period in our analysis for all diseases to be consistent, however, this is not always done in practice. For example, early this year South Sudan waited for seven weeks to declare a cholera epidemic as over, ${ }^{38}$ well beyond twice the 5 day incubation period. Effort to standardize such practices in the future would be welcome. Finally, mortality from these epidemics appears low. While CFR might have been underestimated if deaths occurred after the outbreak was declared over (and therefore not attributed to the outbreak), we believe this possible reporting bias is limited by the existence of the HIS itself that aims capturing both cases and deaths. Such low mortality likely reflected overall good conditions in camps, many of which have been in place for decades.

Despite these limitations, the existence of a standardized health information system is valuable for both the host country and humanitarian actors in their effort to provide evidence-based assistance to displaced population. It allows to assess and monitor health needs, define priorities, as well as monitor the implementation and integration of activities targeting both the host and refugee populations. Efforts to provide disaggregated data at global level as well as additional contextual factors to better understand drivers of health outcomes are welcome.

\section{CONCLUSION}

UNHCR and its partners appear to be successfully containing infectious disease epidemics in refugee camps. Preventive measures addressing WASH and shelter conditions could nevertheless reduce the risk for water and air borne diseases. Vaccination remains a key preventive strategy that needs to be enhanced and adapted to such mobile populations according to context.

\section{ACKNOWLEDGEMENTS}

We thank Dr. Jacob Atem, Postdoctoral Fellow at Johns Hopkins Bloomberg School of Public Health, for his support with population data extraction. 


\section{ETHICS APPROVAL}

This study was deemed as 'non-human subject research' and exempted from ethical clearance by Johns Hopkins Institutional Review Board.

\section{FUNDING}

This work was not supported by any specific funding. MCN is supported by the Delivery Oral Cholera Vaccine (DOVE) Project at Johns Hopkins Bloomberg School of Public Health with grant from the Bill and Melinda Gates Foundation (BMGF).

\section{AUTHORSHIP CONTRIBUTIONS}

CA and PS conceived of the study; CA, VK and AG managed the data; CA conducted the analysis; MN draw the map; CA drafted the first version of the paper; CA, PS and VK contributed to the interpretation of the results; all authors con- tributed to the discussion and approved the final version of the manuscript.

\section{COMPETING INTERESTS}

The authors completed the Unified Competing Interest form at http://www.icmje.org/coi_disclosure.pdf (available upon request from the corresponding authr) and declare no conflicts of interest.

\section{CORRESPONDENCE TO:}

Chiara Altare, $\mathrm{PhD}$

Centre for Humanitarian Health

Department of International Health, Johns Hopkins Bloomberg School of Public Health

21205 Baltimore, Maryland USA

chiara.altare@jhu.edu 


\section{REFERENCES}

1. United Nations High Commissioner for Refugees. Global Trends: Forced Displacement in 2017. Geneva; 2018.

2. Hammer CC, Brainard J, Hunter PR. Risk factors and risk factor cascades for communicable disease outbreaks in complex humanitarian emergencies: a qualitative systematic review. BMJ Glob Health. 2018;3(4):e000647. doi:10.1136/bmigh-2017-000647

3. International Committee of the Red Cross. Health Care in Danger: A Sixteen Country Study. Red Cross; 2011.

4. Wise PH, Barry M. Civil War \& the Global Threat of Pandemics. Daedalus. 2017;146(4):71-84. doi:10.116 2/daed a 00460

5. Medecins sans Frontieres. Refugee Health - An Approach to Emergency Situations. MacMillan; 1997.

6. Toole M, Waldman R. The Public Health Aspects of Complex Emergencies and Refugee Situations. Annu Rev Public Health. 1997;18(1):283-312. doi:10.1146/an nurev.publhealth.18.1.283

7. Spiegel PB, Checchi F, Colombo S, Paik E. Healthcare needs of people affected by conflict: future trends and changing frameworks. Lancet. 2010;375(9711):341-345. doi:10.1016/s0140-6736(0 9) $61873-0$

8. Lam E, McCarthy A, Brennan M. Vaccinepreventable diseases in humanitarian emergencies among refugee and internally-displaced populations. Hum Vaccin Immunother. 2015;11:2627-2636. doi:10.1 $\underline{080 / 21645515.2015 .1096457}$

9. Haskew C, Spiegel P, Tomczyk B, Cornier N, Hering $\mathrm{H}$. A standardized health information system for refugee settings: rationale, challenges and the way forward. Bull World Health Organ. 2010;88(10):792-794. doi:10.2471/blt.09.074096

10. United Nations High Commissioner for Refugees. Health Information System (HIS) - Case Definitions 2010. UNHCR; 2010.

11. United Nations High Commissioner for Refugees. UNHCR Population Statistics - Data - Demographics. Accessed September 28, 2018. http://popstats.unhcr.o rg/en/demographics

12. United Nations High Commissioner for Refugees. Health Information System - Case Definition 2010.
13. World Health Organization. Fact sheets. Accessed September 10, 2018. http://www.who.int/news-room/ fact-sheets

14. Spina A, Lenglet A, Beversluis D, et al. A large outbreak of Hepatitis E virus genotype 1 infection in an urban setting in Chad likely linked to household level transmission factors, 2016-2017. PLOS ONE. 2017;12(11):e0188240. doi:10.1371/journal.pone.018 $\underline{8240}$

15. Guerrero-Latorre L, Carratala A, RodriguezManzano J, Calgua B, Hundesa A, Girones R. Occurrence of water-borne enteric viruses in two settlements based in Eastern Chad: analysis of hepatitis E virus, hepatitis A virus and human adenovirus in water sources. J Water Health. 2011;9(3):515-524. doi:10.2166/wh.2011.126

16. Browne LB, Menkir Z, Kahi V, Maina G, Asnakew $S$, Tubman M. Notes from the field: hepatitis $E$ outbreak among refugees from South Sudan Gambella, Ethiopia, April 2014-January 2015. MMWR Morb Mortal Wkly Rep. 2015;64:537.

17. World Health Organization WPR. Rotavirus. Published 2017. Accessed September 27, 2018. htt p://www.wpro.who.int/mediacentre/factsheets/fs_201 20229/en/

18. Centers for Disease Control and Prevention. The Symptoms of Norovirus. Accessed September 27, 2018. https://www.cdc.gov/norovirus/about/symptom s.html

19. World Health Organization. Cholera : Key facts. Accessed September 27, 2018. https://www.who.int/ne ws-room/fact-sheets/detail/cholera

20. Centers for Disease Control and Prevention. Enterotoxigenic E. coli (ETEC). Accessed September 27, 2018. https://www.cdc.gov/ecoli/etec.html

21. Centers for Diseases Controls and Prevention. RSV Symptoms and Care Respiratory Syncytial Virus. Accessed September 27, 2018. https://www.cdc.gov/rs v/about/symptoms.html

22. Lessler J, Reich NG, Brookmeyer R, Perl TM, Nelson KE, Cummings DA. Incubation periods of acute respiratory viral infections: a systematic review. Lancet Infect Dis. 2009;9(5):291-300. doi:10.1016/s147 3-3099(09)70069-6

23. StataCorp. Stata Statistical Software: Release 15 2017. 


\section{ESRI. ArcGIS Desktop 2016.}

25. United Nations High Commissioner for Refugees. Handbook for Emergencies. 3rd ed. UNHCR; 2013.

26. Navarro-Colorado C, Mahamud A, Burton A, et al. Measles Outbreak Response Among Adolescent and Adult Somali Refugees Displaced by Famine in Kenya and Ethiopia, 2011. J Infect Dis.

2014;210(12):1863-1870. doi:10.1093/infdis/jiu395

27. Kamugisha C, Cairns KL, Akim C. An Outbreak of Measles in Tanzanian Refugee Camps. J Infect Dis. 2003;187(s1):S58-S62. doi:10.1086/368057

28. Kouadio IK, Kamigaki T, Oshitani H. Measles outbreaks in displaced populations: a review of transmission, morbidity and mortality associated factors. BMC Int Health Hum Rights. 2010;10(1):5. do i:10.1186/1472-698x-10-5

29. Deen J, Von Seidlein L, Luquero FJ, et al. The scenario approach for countries considering the addition of oral cholera vaccination in cholera preparedness and control plans. Lancet Infect Dis. 2016;16(1):125-129. doi:10.1016/s1473-3099(15)0029 $\underline{8-4}$

30. Hsiao A, Hall AH, Mogasale V, Quentin W. The health economics of cholera: A systematic review. Vaccine. 2018;36(30):4404-4424. doi:10.1016/j.vaccin e.2018.05.120

31. World Health Organisation. Cholera Vaccines: WHO Position Paper. WHO; 2017.

32. World Health Organization. Meningococcal meningitis. WHO. Published 2018. Accessed October 29, 2018. http://www.who.int/immunization/disease s/meningitis/en/
33. Mustapha MM, Harrison LH. Vaccine prevention of meningococcal disease in Africa: Major advances, remaining challenges. Hum Vaccin Immunother. 2018;14(5):1107-1115. doi:10.1080/21645515.2017.14 $\underline{12020}$

34. Sheikh MA, Makokha F, Hussein AM, Mohamed G, Mach O, Humayun K. Combined use of inactivated and oral poliovirus vaccines in refugee camps and surrounding communities - Kenya, December 2013. MMWR Morb Mortal Wkly Rep. 2014;63:237-241.

35. Biran A, Schmidt WP, Zeleke L, et al. Hygiene and sanitation practices amongst residents of three longterm refugee camps in Thailand, Ethiopia and Kenya. Trop Med Int Health. 2012;17(9):1133-1141. doi:10.11 11/j.1365-3156.2012.03045.x

36. Phillips RM, Vujcic J, Boscoe A, et al. Soap is not enough: handwashing practices and knowledge in refugee camps, Maban County, South Sudan 2015. Confl Health. 2015;9(1):39. doi:10.1186/s13031-015-0 065-2

37. Ziersch A, Due C. A mixed methods systematic review of studies examining the relationship between housing and health for people from refugee and asylum seeking backgrounds. Soc Sci Med. 2018;213:199-219. doi:10.1016/j.socscimed.2018.07.0 $\underline{45}$

38. World Health Organization. South Sudan declares the end of its longest cholera outbreak. WHO Regional Office for Africa. Published 2018. Accessed October 5, 2018. https://afro.who.int/news/south-sud an-declares-end-its-longest-cholera-outbreak 